\title{
Donor-specific and -nonspecific HLA antibodies and outcome post lung transplantation
}

\author{
Stijn E. Verleden ${ }^{1}$, Bart M. Vanaudenaerde ${ }^{1}$, Marie-Paul Emonds ${ }^{2,3}$, \\ Dirk E. Van Raemdonck ${ }^{1}$, Arne P. Neyrinck ${ }^{1}$, Geert M. Verleden ${ }^{1}$ and Robin Vos $^{1}$ \\ Affiliations: ${ }^{1}$ Lung Transplant Unit, Division of Respiratory Disease, Dept of Clinical and Experimental \\ Medicine, KU Leuven, Leuven, Belgium. ${ }^{2}$ HILA laboratory, Rode Kruis Vlaanderen, Mechelen, Belgium. ${ }^{3}$ Dept \\ of Microbiology and Immunology, KU Leuven, Leuven, Belgium.
}

Correspondence: Stijn Verleden, KU Leuven, Lung Transplantation Unit, 49 Herestraat, 3000 Leuven, Belgium. E-mail: stijn.verledenamed.kuleuven.be

@ERSpublications

Persistent donor-specific antibodies are associated with a higher incidence of rCLAD and mortality http://ow.ly/LD5730eFrgl

Cite this article as: Verleden SE, Vanaudenaerde BM, Emonds M-P, et al. Donor-specific and -nonspecific HLA antibodies and outcome post lung transplantation. Eur Respir J 2017; 50: 1701248 [https://doi.org/ 10.1183/13993003.01248-2017].

ABSTRACT Donor-specific antibodies (DSAs) against human leukocyte antigen (HLA) are associated with chronic lung allograft dysfunction (CLAD) and mortality post lung transplantation, but data concerning prevalence, time of onset, persistence and effects on long-term outcome remain scarce.

We assessed the association between HLA antibodies and CLAD-free and graft survival in a cohort of 362 patients. We stratified our analysis according to DSA status, persistence of antibodies and timing of antibodies (pre-transplant, early or late post-transplant).

Within our cohort, 61 (17\%) patients developed DSAs (mostly against HLA-DQ), which was associated with worse CLAD-free and graft survival $(\mathrm{p}<0.0001$ and $\mathrm{p}=0.059$, respectively). Persistent (hazard ratio (HR) 3.386, 95\% CI 1.928-5.948; $\mathrm{p}<0.0001$ ) as well as transient (HR 2.998, 95\% CI 1.406-6.393; p=0.0045) DSAs were associated with shorter CLAD-free survival compared with patients without DSAs. Persistent DSAs (HR 3.071, 95\% CI 1.632-5.778; $\mathrm{p}=0.0005$ ) but not transient DSAs were negatively associated with graft survival compared with patients without DSAs, likely due to the higher incidence of restrictive CLAD. HLA non-DSAs and pre-transplant HLA antibodies had no effect on post-transplant outcome.

We demonstrated an important difference in prognosis between persistent and transient DSAs. Moreover, the observed association between DSAs and restrictive CLAD suggests an overlap between antibody-mediated rejection and restrictive CLAD that needs further investigation.

Received: June 232017 | Accepted after revision: Aug 112017

Support statement: S.E. Verleden is sponsored by grants from the FWO (FWO12G8715N and 1515816N). R. Vos is supported by UZ Leuven (STG15/023) and is a clinical research fellow of the FWO. B.M. Vanaudenaerde is sponsored by KU Leuven (C24/15/030). Funding information for this article has been deposited with the Crossref Funder Registry.

Conflict of interest: None declared.

Copyright @ERS 2017 


\section{Introduction}

Long-term survival after allograft transplantation remains modest across all organs [1]. One of the main reasons for this poor outcome is recognition of the new allograft organ as nonself despite adequate immunosuppression, causing an overt innate and adaptive immune response, ultimately leading to graft fibrosis and loss. The lung is one of the most susceptible organs for this proliferative immune response, leading to chronic lung allograft dysfunction (CLAD). CLAD affects $\sim 50 \%$ of lung transplant patients within 5 years post-transplant, leading to higher morbidity and mortality [2]. In the absence of a specific cause for CLAD, chronic rejection is suspected [3] and recently it has been shown that there are two different phenotypes of chronic rejection: 1) restrictive CLAD (rCLAD) or restrictive allograft syndrome (RAS), characterised by persistent pleuroparenchymal infiltrates, as opposed to 2) an obstructive type (bronchiolitis obliterans syndrome (BOS)), typically showing air trapping [4]. In addition to these clinical differences, patients with rCLAD/RAS have a significantly worse survival compared with BOS patients [4].

In parallel to other solid organ transplants, donor-specific antibodies (DSAs) are linked with later development of chronic rejection in lung transplantation and therefore might play an important role in the pathophysiology of CLAD. Indeed, $10-50 \%$ of patients develop DSAs, increasing the risk of chronic rejection and mortality [5-8]. Recently, a group of experts endorsed by the International Society for Heart and Lung Transplantation defined the presence of DSAs to be typical for antibody-mediated rejection (AMR), in the case of concurrent typical lung histology with or without C4d positivity on biopsy [9]. However, this report also clearly indicated that much more remains to be unravelled about AMR. Moreover, some patients also develop human leukocyte antigen (HLA) antibodies which are not directed against the donor lung allograft; the implications and prognosis for patients with these antibodies are so far unclear.

Therefore, in the present study, we assessed the association between HLA antibodies (either DSAs or non-DSAs) and CLAD and all-cause mortality. We hypothesised that the detection of DSAs would be strongly associated with CLAD (especially rCLAD/RAS) and mortality.

\section{Materials and methods}

Study population

All patients transplanted in UZ Leuven (Leuven, Belgium) between January 1, 2010 and December 31, 2015, and who survived at least 6 months post lung transplantation, were included. Follow-up was recorded until April 1, 2017. For patients undergoing redo transplantation (all for end-stage CLAD), the second transplantation was considered as a separate event for outcome analysis. CLAD-free survival and graft survival (either death or redo transplantation) were the main outcome measurements. When high pre-transplant median fluorescence intensity (MFI) values of HLA antibodies (i.e. $>2000$ ) were detected at the time of investigation of transplant eligibility, prospective virtual HLA cross-matching was performed to avoid specific antigens for transplantation. There was no specific desensitisation protocol for sensitised patients. Only a small number of patients (probable or definite AMR) were treated with plasmapheresis and intravenous immunoglobulin (IVIG), with or without additional administration of rituximab. Our patient management protocol has been described previously [10].

\section{HLA antibody measurement}

Anti-HLA antibodies were detected by Luminex technology according to the institutions' protocol and were classified as either positive or negative. In general, an anti-HLA screening result was considered negative if MFI $<500$ and positive if MFI $\geqslant 500$. A positive screening was followed by the identification of the HLA antibody to define it as DSA or not. Our routine clinical practice includes measurements at day of transplant, hospital discharge, 3, 6, 9, 12, 18 and 24 months post-transplant, and yearly thereafter. In patients with acute or chronic deterioration of graft function, early post-transplant signs of capillaritis or suspicion of AMR, additional measurements are performed. We defined persistent DSAs as the presence of HLA antibodies directed against the same donor HLA locus on at least two separate measurements at least 3 weeks apart. Transient or nonpersistent DSAs were defined as the presence of DSAs only once. The same definitions were applied for HLA non-DSAs.

\section{CLAD definition}

CLAD was defined as an irreversible decline in pulmonary function of at least $20 \%$ compared with the mean of the two best post-transplant values in the absence of confounding factors [11]. We used CLAD as a main end-point in the majority of our analysis; however, these were patients suffering from CLAD with unknown cause only and therefore assumed to be due to chronic rejection. Identifiable causes included pleural fluid, suture stenosis, disease recurrence, etc. When irreversible CLAD without an identifiable cause was found, further subdivision into BOS or rCLAD/RAS was made. rCLAD was defined as a concomitant decline in total lung capacity of at least $10 \%$ in the presence of persistent pleuroparenchymal computed 
tomography (CT) infiltrates [12]. BOS was diagnosed in the remaining patients, and was characterised by an obstructive pulmonary function and air trapping on CT.

\section{Statistics}

Comparison between two continuous variables was performed with the Wilcoxon rank sum test, while Kruskal-Wallis ANOVA was used to compare multiple groups. For comparison of discrete variables, the Chi-squared test was used. The log-rank test was used to compare CLAD-free and all-cause mortality between different groups. The proportional hazards assumption was formally checked by adding the interaction between HLA/DSAs and time to the model, and in none of our analyses was this assumption breached. For univariate analysis, Prism 6.0 (GraphPad, La Jolla, CA, USA) was used. Adjusted analysis was performed using a Cox proportional hazards model using covariates that were hypothesised to be associated with outcome. A p-value $<0.05$ was considered significant.

\section{Results}

\section{Patient cohort}

A total of 383 patients were transplanted during the study period, of whom 20 were excluded because of early ( $<6$ months) post-operative mortality. Median (interquartile range (IQR)) follow-up of this cohort was $3.9(2.3-5.2)$ years. Patient characteristics are shown separately for included and excluded patients in table 1. Briefly, excluded patients were younger and were less likely to be transplanted because of emphysema. Median (IQR) time to death in excluded patients was 71 (21-103) days. Patient death was mostly caused by sepsis/multiorgan failure $(n=7)$, post-surgery $(n=4)$ and by infection $(n=3)$.

\section{Association between DSAs and CLAD and graft survival}

DSAs were detected in 61 out of 363 patients (17\%), and were persistently present in 41 patients $(11 \%)$ and transient in 20 patients (6\%). Median (IQR) time between two positive DSA measurements was 88 (44-191) days. Cumulative DSA occurrence is shown in figure 1a. At 1 year following transplantation, $8 \%$ of patients ever developed DSAs, increasing to $12 \%, 14 \%, 17 \%$ and $19 \%$ at $2,3,4$ and 5 years post-transplant, respectively. Patients with persistent DSAs were slightly younger $(p=0.032)$ and were less likely to be transplanted for emphysema $(\mathrm{p}=0.0022)$. Median time of follow-up in patients with persistent, transient and no DSAs did not differ $(\mathrm{p}=0.58)$.

Comparing patients ever developing DSAs (either persistent or transient) with patients without DSAs demonstrated a significantly worse CLAD-free survival (hazard ratio (HR) 2.86, 95\% CI 2.39-8.13; $\mathrm{p}<0.0001$ ) and a trend for a worse graft survival (HR 1.74, 95\% CI 0.98-3.88; $\mathrm{p}=0.059$ ) (figure $1 \mathrm{~b}$ and $\mathrm{c}$ ). Patients with transient DSAs experienced similar CLAD-free survival compared with patients with persistent DSAs, but there was reduced graft loss, which was comparable to patients without DSAs (figure 2 and patient characteristics in table 1). Given this important difference, we stratified our cohort according

\begin{tabular}{|c|c|c|c|c|c|c|}
\hline Age at transplant years & $56(43-61)$ & $56(46-61)$ & $53(32-60)$ & $45(26-59)$ & $55(45-63)$ & $57(45-59)$ \\
\hline Male & $181(50)$ & $153(51)$ & $28(43)$ & $18(44)$ & $10(50)$ & $14(70)$ \\
\hline \multicolumn{7}{|l|}{ Underlying disease } \\
\hline $\mathrm{CF}+\mathrm{BRECT}$ & $56(16)$ & $46(15)$ & $10(16)$ & $8(20)$ & $2(10)$ & $1(5)$ \\
\hline Redo transplant & $19(5)$ & $14(5)$ & $5(8)$ & $5(12)$ & 0 & $4(20)$ \\
\hline IPAH & $16(4)$ & $14(5)$ & $2(3)$ & $2(0.4)$ & 0 & $1(5)$ \\
\hline Other & $7(2)$ & $4(1)$ & $3(5)$ & $2(0.4)$ & $1(5)$ & $2(10)$ \\
\hline \multicolumn{7}{|l|}{ Type of transplant } \\
\hline SSLTX & 343 (94) & $283(94)$ & $60(98)$ & $40(98)$ & $20(100)$ & $17(85)$ \\
\hline SLTx & $7(2)$ & $7(2)$ & 0 & 0 & 0 & $2(10)$ \\
\hline
\end{tabular}

Data are presented as $\mathrm{n}$, median (interquartile range) or $\mathrm{n}(\%)$. DSA: donor-specific antibody; ILD: interstitial lung disease; CF: cystic fibrosis; BRECT: bronchiectasis; IPAH: idiopathic pulmonary arterial hypertension; SSLTx: sequential single lung transplantation; SLTx: single lung transplantation; LiTx: liver transplantation; HLTx: heart-lung transplantation; KiTx: kidney transplantation. 

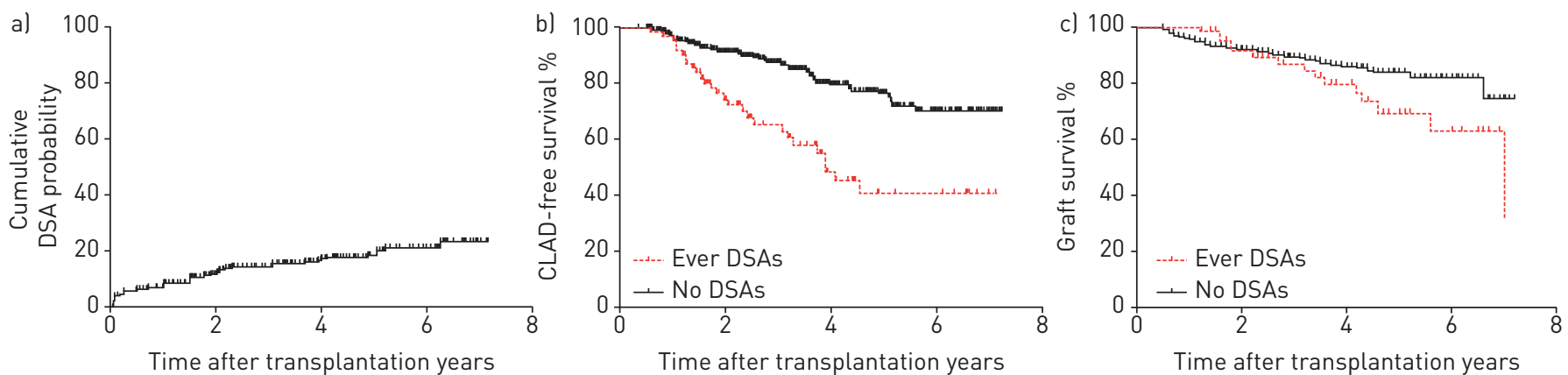

FIGURE 1 Donor-specific antibody (DSA) prevalence and prognosis. CLAD: chronic lung allograft dysfunction. See table 1 for study populations. a) Cumulative DSA occurrence in our patient cohort. At 1 year post-transplant, $8 \%$ of patients ever developed DSAs, increasing to $12 \%, 14 \%, 17 \%$ and $19 \%$ at 2, 3, 4 and 5 years post-transplant, respectively. b) Patients ever developing DSAs demonstrate worse CLAD-free survival ( $<<0.0001$ ); c) patients ever developing DSAs tend to have a worse graft survival ( $p=0.059)$.

to persistent and transient DSAs, and contrasted this to patients never developing DSAs. Time between lung transplantation and positive DSA detection was similar between persistent and transient DSAs (median (IQR) time to DSAs 530 (131-761) versus 62 (22-1280) days; $\mathrm{p}=0.21$ ). Median (IQR) time between first DSA detection and CLAD was 106 (2-541) days. The majority of DSAs were directed against HLA type II antigens, especially HLA-DQ (table 2). Stratification according to type of DSA antibody (HLA-DQ versus other) did not demonstrate a difference in CLAD-free $(\mathrm{p}=0.86)$ or graft survival $(\mathrm{p}=0.39)$. Considering the phenotype of CLAD, it is of interest that $20 \%$ of patients with persistent DSAs developed rCLAD and $27 \%$ BOS, compared with $10 \%$ rCLAD and 30\% BOS in the transient DSA group, and $3 \%$ rCLAD and $16 \%$ BOS in patients without DSAs $(\mathrm{p}=0.034)$. Consequently, graft survival after CLAD diagnosis was lower in patients with persistent DSAs compared with patients with transient or no DSAs $(\mathrm{p}=0.018)$ (figure $2 \mathrm{c}$ ).

Although DSAs have also been associated with an increased rate of acute rejection and lymphocytic bronchiolitis, there was no difference in the incidence of ever acute rejection in our cohort $(\mathrm{p}=0.098)$. However, a difference in the rate of severe acute rejections (grade $\geqslant 2$ ) was seen, with more patients with persistent DSAs experiencing high-grade acute rejection $(\mathrm{p}=0.028)$. There was no difference in the incidence of lymphocytic bronchiolitis $(\mathrm{p}=0.58)$ or high-grade lymphocytic bronchiolitis $(\mathrm{p}=0.35)$ between the different groups (table 2). Similarly, ex vivo lung perfusion (EVLP) has been associated with DSAs post-transplant, but we could not confirm this association in our cohort wherein four out of 24 patients (17\%) who were transplanted following EVLP ever developed DSAs (two persistent), compared with 57 out of 339 patients (17\%) not transplanted on EVLP (39 persistent) $(\mathrm{p}=0.99)$.

We also stratified our analysis according to the time of DSA detection (early: $<3$ months versus late: $>3$ months after transplantation). We observed a similar CLAD-free survival in patients with early DSAs compared with patients with late DSAs, which was inferior to the CLAD-free survival of patients without DSAs $(p<0.0001)$, but we could not observe a difference in graft survival between early DSAs, late DSAs or no DSAs $(\mathrm{p}=0.15)$ (figure $3 \mathrm{a}$ and $\mathrm{b}$ ).
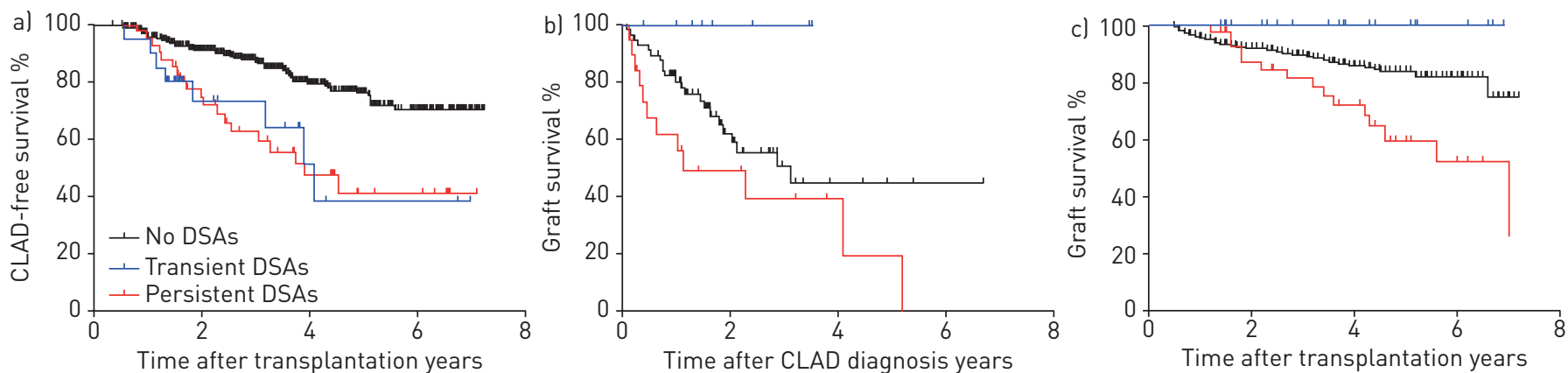

FIGURE 2 Stratification of donor-specific antibodies (DSAs) according to persistence and outcome post-transplant. CLAD: chronic lung allograft dysfunction. See table 1 for study populations. a) CLAD-free survival is similar between patients with persistent and transient DSAs, and is inferior to patients without DSAs ( $p<0.0001$ ); b) graft survival after CLAD diagnosis is lowest in patients with persistent $D S A s(p=0.0008$ ); $c$ ) graft survival is decreased in patients with persistent DSAs but not in patients with transient DSAs, compared with patients without DSAs ( $p=0.018$ ). 
TABLE 2 Primary and secondary outcomes of patients with donor-specific antibodies (DSAs)

\begin{tabular}{lcccc} 
& No DSAs & Ever DSAs & Persistent DSAs & Transient DSAs \\
\hline Patients & 302 & $61(17)$ & $41(11)$ & $20(6)$ \\
Type of DSA & & & & \\
HLA type I & & $2(11)$ & $3(7)$ & $4(20)$ \\
HLA type I+type II & & $31(51)$ & $24(5)$ & $1(5)$ \\
HLA type II (DQ) & & $12(20)$ & $7(17)$ & $9(45)$ \\
HLA type II (DR) & $7(11)$ & $6(15)$ & $5(25)$ \\
HLA type II (DQ+DR) & $57(19)$ & $370(44-765)$ & $530(131-761)$ & $62(22-1280)$ \\
Time to DSAs days & $49(16)$ & $17(28)$ & $19(46)$ & $8(40)$ \\
CLAD incidence & $8(3)$ & $10(16)$ & $8(27)$ & $6(30)$ \\
BOS & $92(30)$ & $24(39)$ & $19(46)$ & $2(10)$ \\
rCLAD & $42(14)$ & $12(20)$ & $11(24)$ & $5(25)$ \\
Ever acute rejection & $120(40)$ & $24(39)$ & $18(44)$ & $1(5)$ \\
Ever acute rejection grade $\geqslant 2$ & $47(16)$ & $13(21)$ & $10(24)$ & $6(30)$ \\
Ever lymphocytic bronchiolitis & & $3(15)$ \\
Ever lymphocytic bronchiolitis grade $\geqslant 2$ & $40)$ &
\end{tabular}

Data are presented as shown as $\mathrm{n}, \mathrm{n}(\%)$ or median (interquartile range). HLA: human leukocyte antigen; CLAD: chronic lung allograft dysfunction; BOS: bronchiolitis obliterans syndrome; rCLAD: restrictive CLAD.

Adjusted analysis including underlying disease, type of transplantation, recipient age and sex demonstrated that either persistent (HR 3.386, 95\% CI 1.928-5.948; $\mathrm{p}<0.0001)$ or transient (HR 2.998, 95\% CI 1.4066.393; $\mathrm{p}=0.0045)$ DSAs were associated with worse CLAD-free survival compared with patients without DSAs. In contrast, only persistent DSAs (HR 3.071, 95\% CI 1.632-5.778; p=0.0005) were associated with worse graft survival. There was no difference in graft survival in patients with transient DSAs and no DSAs ( $\mathrm{p}=0.98$ ). Additional correction in this model for "ever severe acute rejection" or "ever lymphocytic
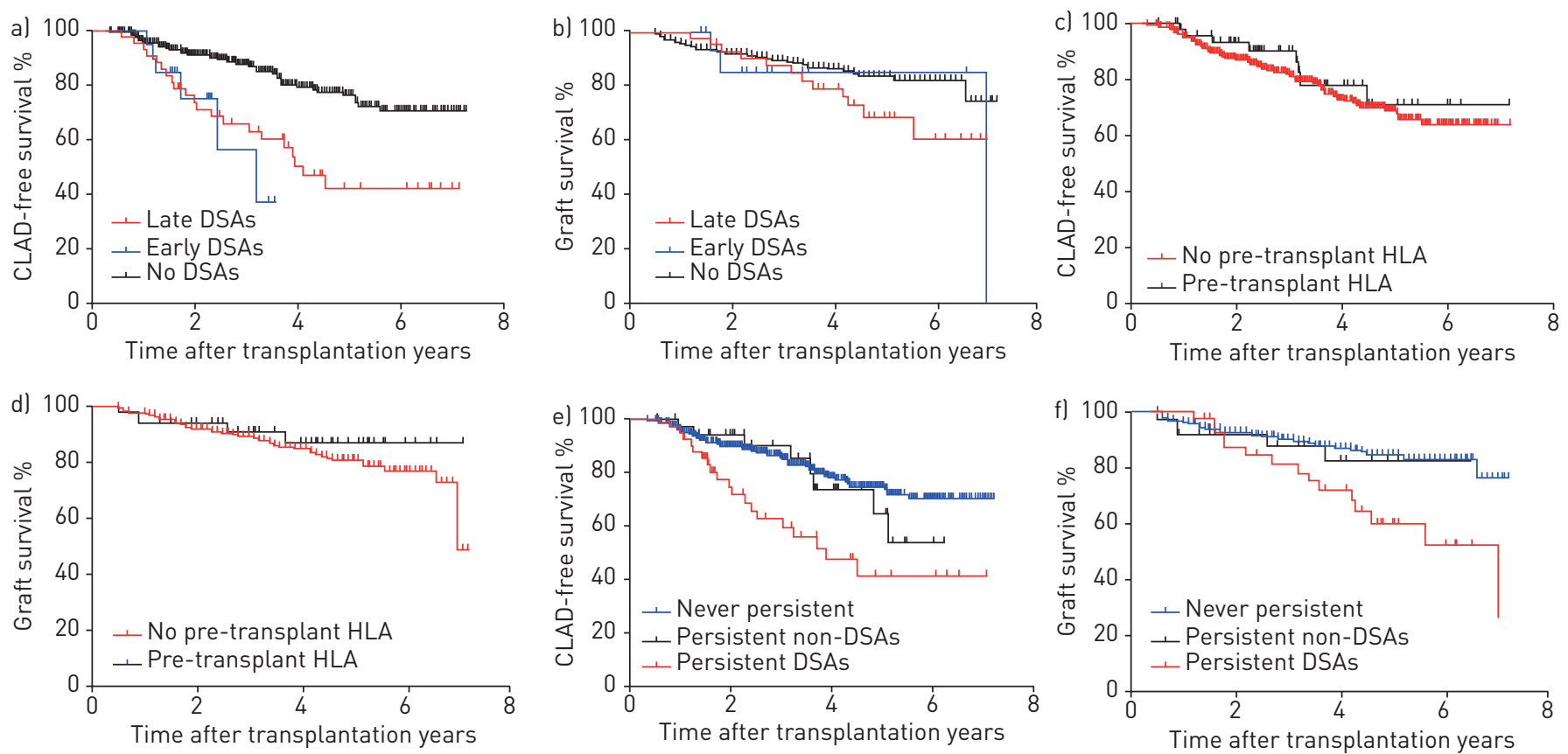

FIGURE 3 Further outcome analysis according to timing of human leukocyte antigen (HLA) donor-specific antibodies (DSAs) and non-DSAs. CLAD: chronic lung allograft dysfunction. a) CLAD-free survival in patients with early DSAs $(n=22)$ is similar to patients with late DSAs ( $n=39$ ) and is inferior compared with patients without DSAs $(n=302)(p<0.0001)$; b) graft survival is similar in patients with early DSAs ( $=22)$, late DSAs ( $=39)$ and no DSAs $(n=302)(p=0.16)$. c) Patients with pre-transplant HLA non-DSAs $(n=48)$ experience a similar CLAD-free survival to patients without $(n=301)(p=0.43)$; d) patients with pre-transplant HLA non-DSAs ( $n=48)$ experience a similar graft survival to patients without ( $n=301)(p=0.37)$. e) CLAD-free survival is similar between patients with persistent HLA non-DSAs ( $n=37$ ) and patients without HLA antibodies (n=285), and is superior to patients with persistent DSAs ( $n=41)(p=0.0005)$; $f$ graft survival is similar between patients with persistent HLA non-DSAs ( $n=37$ ) and patients without HLA antibodies ( $n=285)$, and is superior to patients with persistent DSAs ( $n=41)(p=0.0025)$. 
bronchiolitis" confirmed that the presence of persistent DSAs was independently associated with CLAD-free $(p<0.0001)$ and graft survival $(p=0.0042)$, although severe acute rejection (grade $\geqslant 2)$ was associated with CLAD ( $\mathrm{p}=0.0018)$, but not with graft survival.

\section{Presence of pre-transplant HLA antibodies}

Pre-transplant HLA antibody screening was available in 349 patients (96\%), of which 48 (14\%) had pre-transplant HLA antibodies (HLA class I, $n=9$; HLA class II, $n=23$; HLA class I and II, $n=16$ ). Patients with pre-transplant HLA antibodies were more likely to develop DSAs post-transplant (13 out of 48 patients (27\%) with pre-transplant HLA antibodies developed DSAs versus 46 out of 301 patients (15\%) without pre-transplant HLA antibodies; $\mathrm{p}=0.043$ ). However, there was no association with CLAD-free and graft survival ( $p=0.43$ and $p=0.37$, respectively) (figure $3 c$ and $d$ ). Although patients with pre-transplant HLA antibodies were comparable to patients without pre-transplant HLA antibodies regarding underlying disease $(p=0.071)$, age $(p=0.082)$ and type of transplant $(p=1.00)$, there were significantly more females with pre-transplant HLA antibodies $(\mathrm{p}<0.0001)$.

\section{Post-transplant persistent HLA non-DSAs}

In addition to the patients with persistent DSAs, there was also a significant proportion of patients with post-transplant persistent HLA antibodies not directed against the donor (non-DSAs, $\mathrm{n}=37$ (10\% of the included patient cohort)). The majority of these antibodies were directed against HLA class II antibodies $(\mathrm{n}=17(46 \%))$ or both HLA class I and II $(\mathrm{n}=10(27 \%))$, while the remaining $22 \%$ were directed against HLA class I $(n=10)$. Only 11 out of 27 patients $(41 \%)$ with HLA class II antibodies showed reaction against HLA-DQ, while the majority were directed against HLA-DR ( $\mathrm{n}=15$ (56\%)). Comparing CLAD-free and graft survival between patients with persistent DSAs, persistent non-DSAs and transient or no HLA antibodies demonstrated worse CLAD-free and graft survival in patients with persistent DSAs ( $\mathrm{p}=0.0005$ and $\mathrm{p}=0.0025$, respectively), but could not demonstrate a difference between patients with persistent non-DSAs or patients without HLA antibodies (figure $3 \mathrm{e}$ and $\mathrm{f}$ ).

\section{Discussion}

This is the first study to investigate the influence of DSAs and non-HLA antibodies and long-term outcome in a single cohort, with sufficiently long follow-up. We investigated the association between HLA (both DSAs and non-DSAs) antibodies and outcome post lung transplantation. $17 \%$ of our cohort developed DSAs during their post-transplant follow-up, mostly within the first 2 years and directed against HLA-DQ. Although CLAD-free survival was similar in patients with persistent and transient DSAs, graft loss was significantly higher in patients with persistent DSAs. This is most likely explained by the higher prevalence of rCLAD/RAS. Additionally, patients with either pre-transplant or persistent post-transplant HLA non-DSAs did not experience a worse outcome.

Several reports have already provided evidence that de novo DSAs are associated with a higher incidence of CLAD and a worse outcome following lung transplantation. Remarkably, the rate of DSA incidence is very inconsistent among different centres. While SNYDER et al. [7] reported that $12 \%$ of their patients showed DSAs and Morrell et al. [6] reported 13\%, other studies reported a DSA prevalence of $26 \%$ to $47 \%$ [5, 8]. We do not have a clear explanation for this, although the methodology to measure HLA antibodies varies considerably worldwide. Moreover, our cohort of both donors and recipients is almost exclusively Caucasian, and $<5 \%$ of our cohort was transplanted with EVLP, which might affect DSA development [5].

Patients with persistent DSAs were at higher risk for CLAD and more specifically the rCLAD/RAS phenotype (20\% of patients with persistent DSAs), which further supports the hypothesis that there may be some overlap between AMR and rCLAD/RAS. This is also in line with another publication which showed that patients with AMR exclusively developed RAS [13]. Moreover, immunohistochemical staining of explant lungs of patients re-transplanted for RAS demonstrated a more pronounced adaptive immune response compared with BOS patients, which was evident from the presence of lymphoid follicles occupied by B-cells [14], while immunoglobulins in bronchoalveolar lavage were also elevated at RAS diagnosis [15].

Patients with transient DSAs were also at increased risk of CLAD, but there was no difference in graft loss with all patients currently still being alive. This discrepancy is probably explained by the fact that these patients were less likely to develop rCLAD/RAS. As some previous studies did not make this subdivision into transient and persistent DSAs, and used ever DSAs, this may explain why there was no difference in survival, but only an increased risk for CLAD development [5]. In fact, in our own cohort, pooling the patients with ever DSAs (either persistent or transient) indeed demonstrated a very strong association with CLAD, but only a strong trend for graft loss (figure 1). Therefore, it seems that the persistence of DSAs is of prognostic significance, which needs further investigation. 
There is still scarce evidence about when and how to treat these patients with DSAs. Patients clearing their DSAs after treatment with rituximab and IVIG are less likely to develop BOS and survive longer [16]. Also, treatment with extracorporeal photopheresis may decrease DSAs, antibodies to self-antigens and pro-inflammatory cytokines [17], which can explain its beneficial effect in slowing down the pulmonary function decline in CLAD [18]. Within our cohort, only 15 patients were treated with plasmapheresis and IVIG, of which 10 also received off-label rituximab; their median graft survival after plasmapheresis therapy was 1.5 years. Given that we have only treated patients with deteriorating pulmonary function and mostly following CLAD diagnosis (11 out of 15 patients), and the small proportion of patients treated, our cohort is not suitable to investigate the effect of plasmapheresis or rituximab.

In contrast, patients with HLA non-DSAs did not experience a difference in survival compared with patients without or with transient HLA antibodies. SNYDER et al. [7] investigated the effect of HLA antibodies (either DSAs or non-DSAs) on outcome and demonstrated worse survival in patients with HLA antibodies; however, within our current study, we clearly demonstrate that persistent HLA non-DSAs do not imply a worse outcome. Similarly, the presence of HLA antibodies pre-transplant was not associated with a worse post-transplant prognosis, provided a pre-transplant cross-match was performed to avoid specific HLA antigens. Pre-transplant antibodies were more frequently found in females, most likely due to pregnancyinduced immunisation. These findings confirm the recent study of ZAZUETA et al. [19], which demonstrated no association with pre-transplant HLA antibodies and CLAD nor mortality, although the proportion of patients with HLA antibodies was somewhat lower in our study (14\% versus $30 \%$ ). However, our findings contrast the findings of BRUGIĖRE et al. [20] who showed a significantly worse CLAD-free and overall survival in a small cohort of patients with HLA non-DSAs. As we observed an association between pre-transplant HLA antibodies and later DSA development, we hypothesise that in the presence of pre-transplant HLA antibodies a virtual prospective cross-match or, even better, pre-transplant desensitisation would be useful if an adequate protocol could be developed [21].

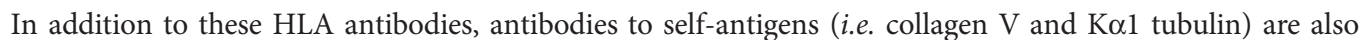
associated with an increased risk of CLAD and death after lung transplantation [22]. However, we could not measure antibodies to self-antigens, which is a limitation of our study. Next, this is a single-centre study, although with a large patient cohort (in fact one of the largest reported thus far) with a median follow-up after transplantation of $\sim 4$ years, which is appropriately long to assess long-term outcome including CLAD; however, it is possible that some end-points were not significant due to a lack of power. Actual MFI numbers were not recorded in our clinical database during the entire study period which did not allow us to stratify DSA positivity according to their MFI, which could be of interest. Persistent DSAs were defined using an interval of 3 weeks, which seems short, as large fluctuations cannot be expected. However, there were several patients who developed de novo DSAs, followed by a rapid progressive form of RAS/rCLAD. We believe that a longer time period (i.e. 6 months) would therefore lead to an underestimation of the prevalence and consequence of DSAs. Indeed, considering an interval $\geqslant 6$ months between at least two positive measurements, only 35 out of 41 patients were persistently positive. However, five out of six patients died due to CLAD within 6 months after DSAs detection.

In conclusion, we confirmed the association between DSAs, CLAD and graft survival after lung transplantation. However, we could demonstrate that, in particular, patients with persistent DSAs have the worst outcome, as they frequently develop RAS. Moreover, we also demonstrated that persistent HLA non-DSAs or pre-transplant HLA antibodies did not impact long-term outcome.

\section{Acknowledgements}

Author contributions: Substantial contributions to the conception or design of the work: S.E. Verleden, G.M. Verleden and R. Vos. Acquisition, analysis or interpretation of data for the work: S.E. Verleden, B.M. Vanaudenaerde, M-P. Emonds, D.E. Van Raemdonck, A.P. Neyrinck, G.M. Verleden and R. Vos. Drafting the work or revising it critically for important intellectual content: all authors. Final approval of the version to be published: all authors.

\section{References}

1 Opelz G, Döhler B, Ruhenstroth A, et al. The Collaborative Transplant Study Registry. Transplant Rev 2013; 27: 43-45.

2 Yusen RD, Edwards LB, Dipchand AI, et al. The Registry of the International Society for Heart and Lung Transplantation: Thirty-third Adult Lung and Heart-Lung Transplant Report - 2016; Focus Theme: Primary Diagnostic Indications for Transplant. J Heart Lung Transplant 2016; 35: 1170-1184.

3 Verleden GM, Raghu G, Meyer KC, et al. A new classification system for chronic lung allograft dysfunction. J Heart Lung Transplant 2014; 33: 127-133.

4 Verleden SE, Ruttens D, Vandermeulen E, et al. Restrictive chronic lung allograft dysfunction: where are we now? $J$ Heart Lung Transplant 2015; 34: 625-630.

5 Tikkanen JM, Singer LG, Kim SJ, et al. De novo DQ donor-specific antibodies are associated with chronic lung allograft dysfunction after lung transplantation. Am J Respir Crit Care Med 2016; 194: 596-606. 
6 Morrell MR, Pilewski JM, Gries CJ, et al. De novo donor-specific HLA antibodies are associated with early and high-grade bronchiolitis obliterans syndrome and death after lung transplantation. J Heart Lung Transplant 2014; 33: $1288-1294$

7 Snyder LD, Wang Z, Chen D-F, et al. Implications for human leukocyte antigen antibodies after lung transplantation: a 10-year experience in 441 patients. Chest 2013; 144: 226-233.

8 Safavi S, Robinson DR, Soresi S, et al. De novo donor HLA-specific antibodies predict development of bronchiolitis obliterans syndrome after lung transplantation. J Heart Lung Transplant 2014; 33: 1273-1281.

9 Levine DJ, Glanville AR, Aboyoun C, et al. Antibody-mediated rejection of the lung: a consensus report of the International Society for Heart and Lung Transplantation. J Heart Lung Transplant 2016; 35: 397-406.

10 Vos R, Ruttens D, Verleden SE, et al. High-dose vitamin D after lung transplantation: a randomized trial. J Heart Lung Transplant 2017; 36: 897-905.

11 Meyer KC, Raghu G, Verleden GM, et al. An international ISHLT/ATS/ERS clinical practice guideline: diagnosis and management of bronchiolitis obliterans syndrome. Eur Respir J 2014; 44: 1479-1503.

12 Sato M, Waddell TK, Wagnetz U, et al. Restrictive allograft syndrome (RAS): a novel form of chronic lung allograft dysfunction. J Heart Lung Transplant 2011; 30: 735-742.

13 Roux A, Bendib Le Lan I, Holifanjaniaina S, et al. Antibody-mediated rejection in lung transplantation: clinical outcomes and donor-specific antibody characteristics. Am J Transplant 2016; 16: 1216-1228.

14 Vandermeulen E, Lammertyn E, Verleden SE, et al. Immunological diversity in phenotypes of chronic lung allograft dysfunction: a comprehensive immunohistochemical analysis. Transpl Int 2017; 30: 134-143.

15 Vandermeulen E, Verleden SE, Bellon $\mathrm{H}$, et al. Humoral immunity in phenotypes of chronic lung allograft dysfunction: a broncho-alveolar lavage fluid analysis. Transpl Immunol 2016; 38: $27-32$.

16 Hachem RR, Yusen RD, Meyers BF, et al. Anti-human leukocyte antigen antibodies and preemptive antibody-directed therapy after lung transplantation. J Heart Lung Transplant 2010; 29: 973-980.

17 Baskaran G, Tiriveedhi V, Ramachandran S, et al. Efficacy of extracorporeal photopheresis in clearance of antibodies to donor-specific and lung-specific antigens in lung transplant recipients. J Heart Lung Transplant 2014; 33: 950-956.

18 Greer M, Dierich M, De Wall C, et al. Phenotyping established chronic lung allograft dysfunction predicts extracorporeal photopheresis response in lung transplant patients. Am J Transplant 2013; 13: 911-918.

19 Zazueta OE, Preston SE, Moniodis A, et al. The presence of pretransplant HLA antibodies does not impact the development of chronic lung allograft dysfunction or CLAD related death. Transplantation 2017; 101: 2207-2212.

20 Brugière $\mathrm{O}$, Suberbielle $\mathrm{C}$, Thabut G, et al. Lung transplantation in patients with pretransplantation donor-specific antibodies detected by Luminex assay. Transplantation 2013; 95: 761-765.

21 Snyder LD, Gray AL, Reynolds JM, et al. Antibody desensitization therapy in highly sensitized lung transplant candidates. Am J Transplant 2014; 14: 849-856.

22 Hachem RR, Tiriveedhi V, Patterson GA, et al. Antibodies to K- $\alpha 1$ tubulin and collagen V are associated with chronic rejection after lung transplantation. Am J Transplant 2012; 12: 2164-2171. 